\title{
Treatment of tuberculous ureteritis. What is the appropriate time for invasive treatment? A case report and review of literature
}

\author{
Özgür Haki Yüksel, Ahmet Ürkmez, Ayhan Verit \\ Fatih Sultan Mehmet Research \& Training Hospital, Dept. of Urology, Istanbul, Turkey.
}

\section{Summary We report a case of isolated distal ureter tuberculosis who presented with irritative voiding symptoms treated with sole medical therapy and discuss the clinical, imaging, diagnostic and therapeutical features. In case of irritative voiding symptoms and radiological presentation of ureteral pathologies, genitourinary tuberculosis should be con- sidered in the differential diagnosis. We believe that medical therapy should be the main option before the invasive procedures.}

KEY WORDS: Ureter tuberculosis; Medical therapy; Invasive treatment.

Submitted 14 June 2014; Accepted 1 August 2014

\section{INTRODUCTION}

Nearly one third of the world's population is estimated to be infected with Mycobacterium tuberculosis.

Genitourinary tuberculosis is not very common but it is considered as a severe form of extra pulmonary tuberculosis. Extra pulmonary tuberculosis accounts for approximately $20 \%$ of cases of active tuberculosis. Only 20 to $30 \%$ of the patients with genitourinary tuberculosis have a history of lung infection (1). Urogenital tuberculosis is characterized by clinical polymorphism. However, the isolated ureteric form is very rare (2). We report a case of isolated distal ureter tuberculosis who presented with irritative voiding symptoms treated with sole medical therapy and discuss the clinical, imaging, diagnostic and therapeutical features.

\section{Case report}

Our case was a 55-year-old woman without significant medical history. The patient consulted us for urinary frequency and microscopic hematuria for the past eight months. The physical examination revealed an exhausted patient. The patient presented with weight loss as $10 \mathrm{~kg}$ in eight months. Laboratory investigations showed appropriate kidney function with a creatinine of $0.97 \mathrm{mg} / \mathrm{dl}$, an inflammatory syndrome with an erythrocyte sedimentation rate of $54 \mathrm{~mm} /$ hour and C-reactive protein of
$2.69 \mathrm{mg} / \mathrm{L}$, whereas the remaining laboratory investigations were unremarkable. The patient then underwent a renal and pelvic ultrasound which showed left hydronephrosis and hydroureter. This examination was completed by a magnetic resonance urography that revealed a left ureterohydronephrosis in the left distal ureter secondary to focal wall thickening (Figure 1). Furthermore, considering the irritative voiding symptoms, we suggested a Koch's bacillus assessment of the patient's urine which resulted strongly positive.

The treatment consisted of antituberculous antibiotics. After 6 months of treatment was observed a decline of hydroureteronephrosis (Figure 2). Mycobacterial culture came to be negative.

\section{Discussion}

The incidence of tuberculosis is estimated as 26 per 100.000 in Turkey. According to the WHO 2006, extrapulmonary tuberculosis rate is $15-25 \%$. In 2005, surveillance of Ministry of Health in Turkey resulted in 20535 cases suffering from tuberculosis, out of them $91.3 \%$ were new cases, $73 \%$ and $27 \%$ were pulmonary and extrapulmonary tuberculosis, respectively. Extrapulmonary tuberculosis cases were genitourinary locations in $4.5 \%$, gastrointestinal and peritoneal locations in $4.5 \%$; intrathoracic lymphadenitis in $5.5 \%$, extrathoracic lymphadenitis in $26 \%$ and pleuric locations in $37 \%$. More rarely bone and central nervous system were affected. Risk factors for extrapulmonary tuberculosis are HIV infection, tumour necrosis factor- $\alpha$ antagonists (e.g. Infliximab), corticosteroids, malignancy, female gender. Being female gender is the unique risk factor for our patient.

Urogenital tubercolosis comprises renal disease, ureteric disease and genital infection. The diagnosis of renal disease is easily missed, as back or flank pain, dysuria or general symptoms occur in only $30 \%$ of patients. Renal tubercolosis is usually unilateral and rarely causes renal failure; the exception is tuberculous interstitial nephritis, which may affect both kidneys. Renal abscesses may destroy the entire renal parenchyma. Pelvo-calyceal involvement may result in thickening of the collecting system; more distally, ureteric fibrosis and stricture formation may cause hydronephrosis, whereas tubercolosis 


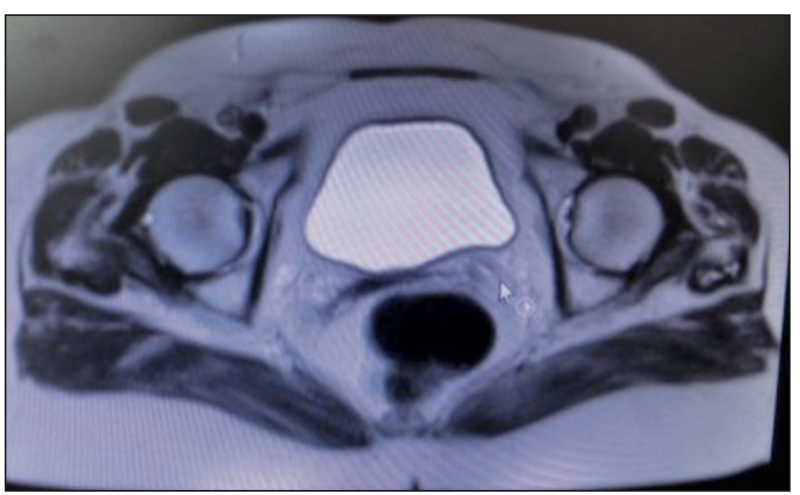

Figure 1.

Magnetic resonanse urography: the left distal ureter secondary to focal wall thickening (marked by the arrow).

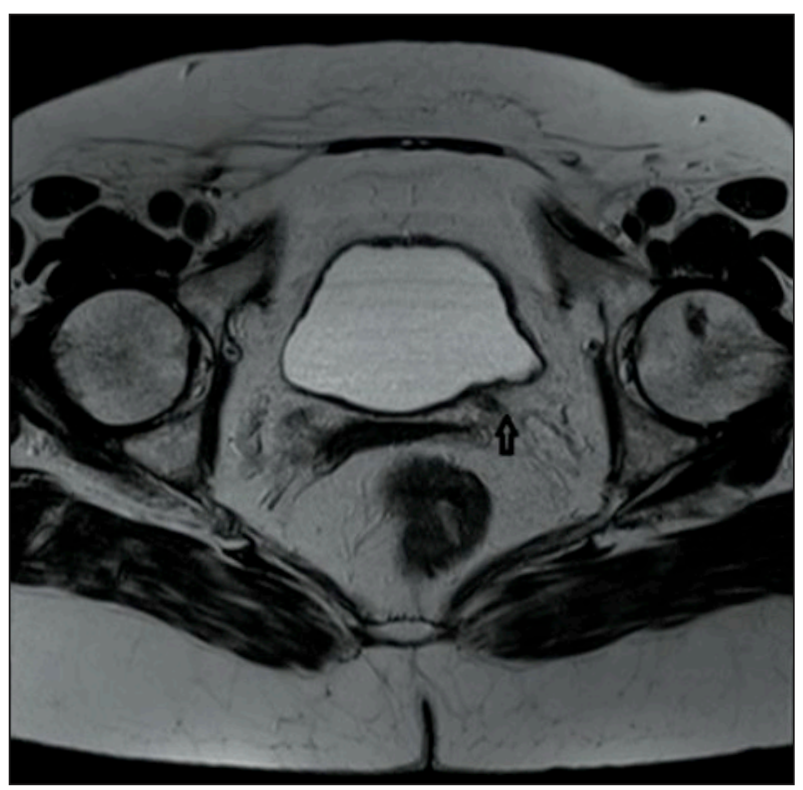

Figure 2.

Magnetic resonanse urography: At the lower end of the left ureter was observed a significant decrease in contrast enhancement (marked by the arrow).

of the bladder wall may lead to fibrosis. Renal biopsy may show granulomatous interstitial nephritis, often with multifocal caseous necrosis. Cystoscopy with biopsies of bladder, ureteric or prostate tissue may also be helpful. The CT/MRG urography and intravenous urography with micturition examinations are designed to make an extended assessment of the urogenital tuberculosis lesions. Imaging or the renal tract may show a characteristically 'beaded' ureter (ureteritis cystica) (3). This is probably due to an extending fibro-inflammatory process with thickening of the ureteral wall that could be confused with a ureteral tumor in evaluation of imaging (4). Urogenital tuberculosis is characterized by varied clinical symptoms (5). Ureteral localization was always described as secondary to renal disease because it represents the extension of mucosal lesions from the kidney (6).

The case reported here did not reveal any visible renal impairment by imaging exploration except hydroureteronephrosis.

Endoscopy must always be performed with the patient under general anesthesia with a muscle relaxant to reduce the risk of hemorrhage. The phase of bladder filling should be performed under direct vision. Bladder biopsy is contraindicated in the presence of acute tuberculous cystitis (7). Indications for ureteroscopy are rare but renal tuberculosis should be included in the differential diagnosis of lateralizing hematuria, especially in the absence of an obvious cause for the bleeding. In this case direct culture of urine from the renal pelvis may have more sensitivity than culture of voided urine (8).

The confirmation of the diagnosis is based on assessing microscopically the presence Koch's bacilli in the urine by direct testing for alcohol-acid-resistant bacillus. The Koch's bacillus culture requires a long time for obtaining the final results as long as eight weeks. The identification of Koch's bacillus using polymerase chain reaction is faster and takes 24 to 48 hours, but with a sensitivity reduced to $48.5 \%$ (9).

According to the $\mathrm{WHO}$, the antituberculous drug treatment is based on an initial 2 months intensive phase of treatment with three or four drugs (rifampicin, isoniazid, pyrazinamide, etambutol or streptomycin) to destroy almost all tuberculous bacilli. This is followed by a 4 months manteinance phase with only two drug mostly rifampicin and soniazid (10).

The most common site for tuberculous stricture is the ureterovesical junction. Uretheral strictures may develop in more than $50 \%$ of patients with renal involvement (11). Strictures of the lower end of the ureter, which can either be managed medically or surgically, occur in approximately $9 \%$ of patients. If obstruction at the lower end of the ureter is present at the start of chemotherapy careful observation is required. These strictures may result from edema and they respond to chemotherapy. The patient should receive chemotherapy and should be monitored by intravenous urograms at weekly intervals. Corticosteroids can be added to chemotherapy if there is deterioration or no improvement after 3 weeks. If there is still deterioration or no improvement after a 6 week period, surgical reimplantation should be carried out if an initial attempt of dilatation has failed. Double J ureteral catheter drainage may be used during this period for assessing the efficacy of medical therapy. Early ureteral stenting or PCN (percutaneous nephrostomy) in patients with tuberculous ureteral strictures may increase the opportunity for later reconstructive surgery and decrease the likelihood of renal loss (12). In all other situations, patients should have at least 4 weeks of extensive chemotherapy before surgery (7).

The overall incidence of surgical management of genitourinary tuberculosis in the past 20 years was reported to be about $0.5 \%$ of all urological surgical procedures (13). Although chemotherapy is the mainstay of treatment, ablative surgery as a first-line management may be unavoidable for sepsis or abscesses (14). Medical treatment is the first-line therapy in genitourinary tuberculosis. Both radical and reconstructive surgery should be carried out in the first 2 months of intensive chemotherapy (15). The duration of medical therapy has been 
reduced to 6 months in uncomplicated cases. Only in complicated cases (recurrences of tuberculosis, immunosuppression and HIV/AIDS) a 9 to 12 month therapy is necessary (6).

In conclusion, in the presence of irritative voiding symptoms and radiological imaging showing a pathological ureter, genitourinary tuberculosis should be considered in the differential diagnosis. We believe that medical therapy should be the main option before the invasive procedures.

\section{REFERENCES}

1. World Health Organization (WHO) (2010) Global Tuberculosis Control 2010: Epidemiology, Strategy, Financing. WHO/HTM/TB 2010. 7.

2. Matos MJ, Bacelar MT, Pinto P, Ramos I. Genitourinary tuberculosis. Euro J Radiol. 2005; 55:181.

3. Figueiredo AA, Lucon AM, Arvellos AN, et al. A better understanding of urogenital tuberculosis pathophysiology based on radiological findings. Eur J Radiol. 2010; 76:246.

4. Dhar NB, Angermeier KW. Idiopathic ureteral strictures without evidence of malignancy. Urology. 2004; 64:377.

5. El Khader K, Lrhorfi MH, el Fassi J, et al. Tuberculose urogénitale expérience de 10 ans. Prog Urol. 2001; 11:62.
6. Cek M, Lenk S, Naber KG, et al. Members of the Urinary Tract Infection (UTI) Working Group of the European Association of Urology (EAU) Guidelines Office. EAU guidelines for the management of genitourinary tuberculosis. Euro Urol. 2005; 48:353

7. Warren D, Johnson JR, Johnson CW, Franklin C. Lowe: Genitourinary TuberculosisCampbell's Urology. 8th ed. Saunders; 2002

8. Chan SW, Shalhav AL, Clayman RV. Renal tuberculosis presenting as lateralizing hematuria diagnosis by ureteronephroscopy and selective upper tract urine culture. Endourol. 1998; 12:363.

9. Bouchikhi AA, Amiroune D, Tazi MF, et al. Pseudotumoral tuberculous ureteritis: a case report. J Med Case Rep. 2013; 15:45.

10. World Health Organization (WHO) Anti-tuberculosis drug resistance in the world. Report No. 4. WHO/HTM/TB/2008.394.

11. Allen FJ, deKock ML. Genito-urinary tuberculosis-experience with 52 urology in patients. S Afr Med J. 1993; 83:903.

12. Shin KY, Park HJ, Lee JJ, et al. Role of early endourologic management of tuberculous ureteral strictures. J Endourol. 2002; 16:755.

13. Rizzo M, Ponchietti R, Di Loro F, et al. Twenty-years experience on genitourinary tuberculosis. Arch Ital Urol Androl. 2004; 76:83.

14. Carl P, Stark L. Indications for surgical management of genitourinary tuberculosis. World J Surg. 1997; 21:505.

15. Gow JG. Tuberculosis: genitourinary tuberculosis. Br J Hosp Med. 1979; 22:556.

\section{Correspondence}

Özgür Haki Yüksel, MD (Corresponding Author) ozgurhaki@gmail.com

Ahmet Ürkmez, MD

Ayhan Verit, MD, Prof

Fatih Sultan Mehmet Research and Training Hospital, Dept. of Urology,

Icerenkoy/Atasehir Tr- 34752 Istanbul, Turkey 